\title{
I barbari, la letteratura e i nuovi media (1990-2007)
}

\section{Federico Pellizzi}

\section{OpenEdition}

\section{Journals}

\section{Edizione digitale}

URL: http://journals.openedition.org/cei/138

DOI: $10.4000 /$ cei. 138

ISSN: 2260-779X

\section{Editore}

UGA Éditions/Université Grenoble Alpes

\section{Edizione cartacea}

Data di pubblicazione: 15 juin 2010

Paginazione: 195-213

ISBN: 978-2-84310-168-7

ISSN: $1770-9571$

Notizia bibliografica digitale

Federico Pellizzi, «l barbari, la letteratura e i nuovi media (1990-2007)», Cahiers d'études italiennes [Online], 11 | 2010, online dal 15 décembre 2011, consultato il 27 mars 2021. URL: http:// journals.openedition.org/cei/138; DOl: https://doi.org/10.4000/cei.138 


\title{
I BARBARI, LA LETTERATURA E I NUOVI MEDIA
}

(1990-2007)

\author{
Federico Pellizzi \\ Université de Bologne
}

Il primo marzo del 2006 apparve sul quotidiano La Repubblica un intervento di Alessandro Baricco, dal titolo "Cari critici leggetemi e dopo stroncate», con finestra in prima pagina, che accusava i critici «mandarini» - e nello specifico Pietro Citati e Giulio Ferroni, di liquidare i suoi libri con delle frasi sferzanti invece di farne, semmai, una seria lettura critica ${ }^{\mathrm{I}}$. $\mathrm{Ne}$ nacque un dibattito, sui quotidiani e in rete, che coinvolse molti altri critici e scrittori. Solo su Repubblica intervennero, tra gli altri, lo stesso Ferroni, Nello Ajello, Carla Benedetti, Loredana Lipperini, Pietrangelo Buttafuoco, Stefano Bartezzaghi, Antonio Scurati, Carlo Lucarelli, Edmondo Berselli, e Antonio Moresco; numerosi furono anche gli intervistati, da Roger Silvers, a Edoardo Sanguineti, a Mario Lavagetto ${ }^{2}$.

Sebbene l'argomento della disputa fosse ufficialmente la deontologia della critica militante, ne uscirono a mio parere degli interessantissimi scorci non solo sulla realtà letteraria e culturale italiana, ma anche, in particolare, sul rapporto tra letteratura e media. Tra le righe dei vari interventi, di là dal riproporsi, in certi casi, di viete questioni ${ }^{3}$, emergeva perfino l'impressione che in gioco ci fossero diverse e incompatibili concezioni della letteratura, del nostro tempo e della nostra società.

I. La Repubblica, I marzo 2006, pp. I, 44-45.

2. La Repubblica, 2 marzo 2006, pp. 46-47; 3 marzo 2006, pp. 56-57; 8 marzo 2006, p. 50.

3. Questioni che pure danno da pensare, come il venir meno della funzione della recensione autorevole, su cui si dibatte da anni, ma che spesso sono ridotte a lamentosi cliché (la morte vera o presunta della critica, la sua subalternità al mercato e all'industria editoriale), o ridicolizzate proponendo - come nelle pagine de La Repubblica che accompagnavano questo dibattito - le regole d'oro del recensore, e altre amenità, come la rappresentazione arborea di un'improbabile «mappa del potere letterario» italiano a mezza pagina (La Repubblica, 3 marzo 2006, pp. 56-57). 
Che altro ci vuole per scatenare finalmente una bella, approfondita discussione? Invece niente. Il fatto è che tali concezioni non erano dichiarate ed esposte, bensì trasparivano qui e là. Non erano messe al centro della discussione, ma solo accennate o sottintese, e comunque non raccolte dai contendenti, travisate o eluse, obliterate da altre battute icastiche o ironiche.

Eppure nell'intervento di Baricco, misurato e abilmente costruito, nonché punteggiato di apparenti considerazioni autocritiche e di professioni di umiltà ("per quello che ne capisco, i miei libri saranno presto dimenticati, e andrà già bene se rimarrà qualche memoria di loro per i film che ci avranno girato su. Così va il mondo. E comunque, lo so, i grandi scrittori, oggi, sono altri»), gli accenni erano abbastanza espliciti:

Ma quale arroganza intellettuale può indurre a pensare che non sia utile capire una degenerazione del genere [Baricco allude qui alla diffusione dei suoi libri], e magari spiegarla a chi non ha strumenti per comprenderla? Come si fa a non intuire che i miei libri sono poca cosa, ma lì i lettori ci trovano qualcosa che allude a un'idea differente di libro, di narrazione scritta, di emozione della lettura? Perché non provate a pensare che esattamente quello - una nuova, sgradevole, discutibile idea di piacere letterario - è il virus che è già in circolo nel sistema sanguigno dei lettori, e che magari molta gente avrebbe bisogno da voi che gli spiegaste cos'è questo impensabile che sta arrivando, e questa apparente apocalisse che li sta seducendo? Non sarà per caso che la riflessione nel campo aperto del futuro vi impaurisce, e che preferite raccogliere consensi declinando da maestri mappe di un vecchio mondo che ormai conosciamo a memoria, rifiutandovi di prendere atto che altri mondi sono stati scoperti, e la gente già ci sta vivendo? Se quei mondi vi fanno ribrezzo, e la migrazione massiccia verso di loro vi scandalizza, non sarebbe esattamente vostro degnissimo compito il dirlo? Ma dirlo con l'intelligenza e la sapienza che la gente vi riconosce, non con quelle battutine, please. ${ }^{4}$

E poco prima aveva scritto:

Vi fa schifo che uno adatti l'Iliade per una lettura pubblica e lo faccia in quel modo? Forse è il caso di dirlo in maniera un po' più argomentata e profonda, chissà che ci scappi una riflessione utile sul nostro rapporto con il passato, chissà che non vi balugini l'idea che una nuova civiltà sta arrivando, in cui l'uso del passato non avrà niente a che fare con il vostro collezionismo raffinato e inutile. ${ }^{5}$

Di là dagli obiettivi polemici diretti (la protesta per le battute liquidatorie), forse si può essere sviati anche dalla retorica baricchiana, e prendere queste affermazioni, vaghe e spaventose, come un tipico esempio di quell'enfasi illusionistica che gli rimprovera Ferroni. Non giova nemmeno

4. La Repubblica, I marzo 2006, pp. 44-45, corsivi miei.

5. Ivi, p. 44, corsivi miei. 
la ricaduta in ciò che può sembrare una sorta di megalomania egocentrica, benché maliziosa (la pretesa di incarnare il nuovo, di rispondere al bisogno di un nuovo tipo di piacere letterario, o addirittura di essere - sotto sotto - il virus che lo diffonde) ${ }^{6}$.

Eppure Baricco pone una questione molto chiara, parla di un cambiamento di sensibilità, di uno scarto nell'immaginario, del crescere di nuovi lettori. È una questione che va molto oltre l'obiettivo polemico circoscritto delle istituzioni letterarie italiane. Nonostante ciò, negli interventi dei giorni successivi, quasi nessuno raccoglie e discute questo spunto. Solo uno scrittore che sta agli antipodi di Baricco, Antonio Moresco, cerca di allargare il quadro, dando conto di un proprio coinvolgimento emozionale e di un ritrovato interesse per gli ultimi libri di Baricco:

In Baricco, e in particolare nei suoi ultimi libri, è sempre più incombente la morte. I suoi personaggi cercano di oltrepassarla inscrivendola in un gesto che li renda mitici e unici. Una dimensione sentimentale, ultraromantica e pop, che è l'altra faccia di quella pubblicitaria di questa epoca [...]. Qui, in questa megalomania infantile, sono trasportato nell'indistinzione tra dimensione pubblicitaria e mitizzazione, qui c’è qualcosa che mi avvicina di più a quanto sta succedendo realmente nell'immaginario di questi anni. Questa vita è sempre più al cospetto della morte [...]. Il bambino fortunato ha visto che dietro la maschera pubblicitaria della fortuna c'è la morte e ne è rimasto turbato e sconvolto. Quella che incombe su tutti e quella che incombe anche su di lui come uomo e come scrittore. Ma è esattamente quello che c'è dietro la maschera della nostra epoca e del nostro mondo. Cerca di esorcizzarla e di sublimarla mediante il suo sogno pubblicitario e infantile. ${ }^{7}$

Nessun altro dei partecipanti al dibattito dice qualcosa sull'immaginario del nostro tempo, o sui cambiamenti a cui accenna Baricco. Tutti si limitano a commentare, solidarizzando o dissentendo, le regole e i costumi del sistema letterario nostrano.

Ferroni, nella sua risposta, fa notare di avere già scritto ampiamente di Baricco e perfino recensito - stroncando senza battutine - l'ultimo suo romanzo, Questa storia ${ }^{8}$. Sanguineti afferma che «la pretesa mossa dallo scrittore in nome delle alte tirature e delle traduzioni in tutto il

6. Il virus è dopotutto uno spauracchio relativamente recente, diffusosi particolarmente negli anni Novanta, che ha sostituito nel nostro immaginario ogni spettro che si aggiri per il mondo. Stephan Jay Gould proponeva sul finire degli anni Novanta l'infezione virale come metafora biologica per il cambiamento culturale (S. J. Gould, «Pluralismo darwiniano», La rivista dei libri, n. II, novembre 1997, p. 28). La metafora ricorre anche in D. Brolli, ideatore dei "giovani cannibali" e curatore di altre antologie significative degli anni Novanta, del cyberpunk e del post-cyberpunk (come ad esempio Cavalieri elettrici, Roma-Napoli, Theoria, I995; Cuori elettrici. Antologia essenziale del cyberpunk, Torino, Einaudi, 1996). La cyber-mania è presa di mira dal narratore di Occhi sulla graticola di T. Scarpa, di cui si dirà in seguito.

7. La Repubblica, 8 marzo 2006, p. 50, corsivo mio.

8. La Repubblica, 2 marzo 2006, p. 47. 
mondo» di essere recensito è ingiustificata: «Una critica seria può serenamente disinteressarsi d'un libro molto venduto e liquidarlo con una semplice battuta.» ${ }^{9}$ Carla Benedetti, che parteggia per Baricco, stigmatizza la piccineria e la «superficialità grottesca di certo giornalismo culturale», e denuncia la schizofrenia di un sistema che promuove i libri di plastica e poi li snobba ${ }^{\mathrm{IO}}$. Lucarelli, che $-\mathrm{da}$ volto televisivo e scrittore considerato da alcuni critici "paraletterario» - si sente vicino a Baricco, vagheggia una comunità urbana e appassionata di critici, lettori, e scrittori dove non prevalgano le politiche editoriali e le questioni personali ${ }^{\text {II }}$. Dai blog, dove la polemica è rilanciata, emergono molti commenti, spesso riassumibili in questa forma: «non mi piace la scrittura di Baricco, ma contro i critici ha ragione.» ${ }^{\text {I2 }}$

Nessuno sembra raccogliere questo accenno ad «altri mondi» o a paventate apocalissi, questo invito a parlare di cambiamenti che nessuno conosce ma tutti percepiscono. Ma la domanda che ci dovremmo fare a questo punto è: perché nessuno risponde a Baricco? Perché quasi nessuno coglie il contesto del discorso di Baricco? Lasciamo in sospeso per ora i quesiti.

A ben guardare, tuttavia, le enunciazioni in forma di domande multiple di Baricco che ho riportato sono l'anticipazione, il nucleo originario di un'impresa che forse era già in cantiere, o forse si è creata proprio a partire da quell'intervento: cioè la stesura, sempre sulle pagine de $L a$ Repubblica, di un libro-saggio a puntate che doveva avere come titolo La mutazione e che assume il titolo definitivo I barbari. La pubblicazione inizia il 12 maggio e prosegue per trenta puntate, con due interruzioni, fino al 2I ottobre 2006. Si tratta di un esperimento singolare, unico, un "tentativo - scrive Baricco nella prima puntata - di pensare: scrivendo». La sollecitazione a scrivere il libro è data proprio da quella percezione di cambiamento e dal desiderio di capire quel che sta succedendo: "Dovendo riassumere, direi questo: tutti a sentire nell'aria, un'incomprensibile apocalisse imminente: stanno arrivando i barbari.» ${ }^{13}$

Attraverso le puntate, che si dipanano attraverso le amplificazioni, le contrazioni, le interpunzioni enfatiche, le associazioni simboliche tipiche della prosa di Baricco, l'autore cerca di fare il ritratto a questi barbari, di

\footnotetext{
9. Ibidem.

Io. Ibidem.

II. La Repubblica, 3 marzo 2006, pp. 56-57.

I2. Ne dà conto Loredana Lipperini, "Gli internauti: "ha ragione Alessandro" ", La Repubblica, 2 marzo 2006, p. 47.

13. Alessandro Baricco, I barbari, Roma, La biblioteca di Reppubblica, 2006, p. I2. [B].
} 
capire chi siano, che cosa pensino e come si comportino. Non c'è il tempo per seguire tutte le tappe e per esaminare in dettaglio gli elementi che Baricco assegna a quella che si potrebbe chiamare la "logica barbarica" dei nostri tempi. Possiamo soffermarci su alcuni aspetti. Di sicuro si intravedono, dietro alla logica barbarica, alcuni tratti della poetica di Baricco: la necessità di trovare una lingua semplice ed emozionale, l'attenzione ai ritmi e alle modalità della comunicazione di massa; inoltre si riconoscono alcuni dogmi dell'immaginario mediatico: la perdita dell'esperienza, la sequenzialità, l'orizzontalità, lo stare alla superficie (ossia l'eclisse della profondità), la velocità, la connessione, la leggerezza. Così scomposti e messi a nudo, fuori dall'impalcatura di Baricco, questi concetti sembrano poca cosa. Ma qui conta anche il percorso: Baricco comincia a descrivere i barbari distanziandosi da loro, esseri mutanti dotati di branchie; poi gli effetti della mutazione cominciano a intravedersi anche sul dorso di Baricco; e infine siamo coinvolti tutti, anche il lettore, e si scopre che i barbari siamo proprio noi.

I barbari sono come pesci mutanti dotati di branchie che concepiscono il senso solo come movimento tra sistemi passanti. Hanno un'idea di libro che non è più quella tradizionale: «i barbari tendono a leggere solo i libri le cui istruzioni per l'uso sono date in posti che $\mathrm{NON}$ sono libri» (B, p. 9I): è in fondo una percezione ipermediale del libro, di un libro che vive non più soltanto in rapporto ad altri libri, ma che viene a far parte di un circuito più ampio. E quello a cui stiamo assistendo oggi, anche sul piano editoriale, è sotto gli occhi e i sensi di tutti anche se sembra troppo banale parlarne: «I barbari usano il libro per completare sequenze di senso che sono generate altrove.» (B, p. 9o.)

Giulio Ferroni, che aveva promesso, nella sua risposta all'intervento di Baricco su La Repubblica, di non occuparsi più di lui, torna invece ad occuparsene in un pamphlet che esce prontamente il 25 agosto $2006^{14}$, quando le puntate sono ancora in pieno svolgimento. È, naturalmente, una stroncatura:

Egli vi dice che il banale è essenziale, che la mediocrità è distinzione, che il facile è difficile, e per converso che l'essenziale è banale, che la distinzione è mediocrità, che il difficile è facile: ma, nel modo in cui ve lo dice, nel percorso attraverso cui giunge a rivelarvelo, sancisce continuamente il proprio essere dalla parte di un'essenzialità,

14. Giulio Ferroni, Massimo Onofri, Filippo La Porta e Alfonso Berardinelli, Sul banco dei cattivi. A proposito di Baricco e di altri scrittori alla moda, Roma, Donzelli, 2006. Il saggio di G. Ferroni, Profondità di superficie, è alle pp. 9-3I. 
di una distinzione, di una difficoltà, di qualcosa che comunque resta inafferrabile e segreto, che dovete considerare di sua suprema competenza, dono esclusivo del suo essere artista, tanto più sfuggente quanto più per il resto lui stesso vi mostra che l'esclusività è facilmente moltiplicabile, che tutto può arrivare in superficie, che tutto può scorrere sul nastro del presente. ${ }^{\text {Is }}$

Dal pamphlet si ricava che, a Ferroni, Baricco proprio non piace; ma si ricava anche un altro elemento: la critica di Ferroni si colloca esclusivamente su un piano stilistico-letterario e idiosincratico: Ferroni non si concentra su ciò che Baricco dice sul mondo, ma su come il suo modo di dirlo crei una rappresentazione del mondo «da cui sembra esclusa - dice Ferroni - ogni contraddizione e ogni vera conflittualità» ${ }^{16}$. D'accordo, solo che Baricco sta proprio esibendo un conflitto e una contraddizione: stanno arrivando i barbari. Vi sembra poco? Non solo, ma colloca anche storicamente questa frattura: l'antecedente paradigmatico è un passo di Goffredo Parise del 1974, il quale, parlando di Piovene, colloca quest'ultimo insieme a Gadda, Montale, Moravia, Comisso in quella che definisce «last generation», mentre colloca se stesso in un tempo nuovo (B, p. 95). E qual è la ragione di questo scarto? Ecco la spiegazione di Parise, citato da Baricco: «Piovene, come Montale e Moravia e al contrario di noi, aveva vissuto un certo numero di anni in cui la parola scritta fu espressione molto prima che comunicazione.» (B, p. 96, corsivi miei.) Tornerò tra breve su questa frase. Baricco la commenta in modo radicale: "Come intuì Parise, non si trattava di una semplice variazione allo statuto di un'arte: ne era la fine» (B, p. 96). Che cosa è successo alla letteratura da allora? Sempre più ha dovuto adottare la «lingua del mondo» la «lingua dell'Impero». «Il primo libro di qualità - dice Baricco - a intuire questa svolta, e a cavalcarla, [è] stato Il nome della Rosa, di Umberto Eco (I980, bestseller planetario)» (B, p. 98). Ferroni commenta: «tout se tient.» ${ }^{17} \mathrm{Ma}$ dopo queste tappe di avvicinamento, il punto di svolta è indicato chiaramente da Baricco nelle puntate successive, dedicate al motore di ricerca Google, pubblicate tra il I3 e il 25 luglio dopo una pausa di quattordici giorni.

Non posso soffermarmi ora dettagliatamente su quei capitoli. Basti dire che Baricco paragona Google all'invenzione della stampa: è un sistema che, come la stampa, «modifica il sapere a propria immagine». Aggiunge Baricco:

I5. Giulio Ferroni, Profondità di superficie, cit., p. I3.

I6. Ivi, p. I9.

I7. Ivi, p. 22. 
se qui parliamo di Google non stiamo parlando di una robetta curiosa o di un'esperienza come un'altra, tipo il vino o il calcio [di cui aveva parlato nella prima serie di puntate]. Google non ha nemmeno dieci anni di vita, ed è già nel cuore della nostra civiltà: se tu lo spii, non stai visitando un villaggio saccheggiato dai barbari: sei nel loro accampamento, nella loro capitale, nel palazzo imperiale.

E, indovinate: dove Ferroni decide di interrompere definitivamente la lettura del saggio-feuilleton di Baricco? Proprio qui, proprio dove quest'ultimo comincia a parlare di Google. A me sembra molto indicativo. Il vero impensabile è la rete, il vero perturbante è il digitale. Perché la sua portata è enorme e di lungo corso: tutte le rivoluzioni annunciate, legate a questo o a quell'aspetto particolare si sono rivelate fole: ciò che cambia è il sistema, la civiltà. Sarà anche questa, come lo sviluppo della stampa, una «rivoluzione inavvertita» (il termine è di Elizabeth Eisenstein, storica dei cambiamenti sociali dell'età della stampa), che forse solo i nostri bisnipoti cominceranno a comprendere davvero?

Non sappiamo, ma possiamo tornare a chiederci perché nessuno risponda a Baricco. A questo punto possiamo ipotizzare che probabilmente è una forma di scotoma, di rimozione. Le mutazioni sono dolorose e spaventose.

Si afferma che siamo nell' «era della televisione» ${ }^{18}$ ma, viene da dire, solo di questo? Una televisione - per altro spesso totalmente incompresa - che diviene paradigma addirittura di un'era. Si generalizza un'idea molto circoscritta di televisione (una tv giudicata soprattutto per i suoi contenuti espliciti prevalenti - violenza, stupidità, ecc. - o indicata come la causa della presente corruzione dei costumi - consumismo, manipolazione del consenso, falsificazione del reale, ecc. - e non considerata per le ben più vaste modificazioni percettive ed esistenziali che ha introdotto, esibito o accentuato). E di questa presunta televisione si dà per scontata la centralità: un monoblocco verso cui tutto dovrebbe convergere (il vecchio mito della "convergenza digitale» negli anni Novanta aveva come modello economico produttivo e di consumo proprio la televisione di allora) ${ }^{19}$. Ma anche questo atteggiamento inerziale sembra sintomo di rimozione.

I8. Jean-Jacques Wunenburger, L'homme à l'âge de la télévision, Paris, PUF, 200o; tr. it. L'uomo nell'era della televisione, Napoli, Ipermedium libri, 2005; Giovanni Sartori, Homo videns. Televisione e post-pensiero, Bari, Laterza, 2000; e, da ultimo, Antonio Scurati, La letteratura dell'inesperienza. Scrivere romanzi al tempo della televisione, Milano, Bompiani, 2006. Indizio di come sia particolarmente viva in Italia l'ossessione per la televisione è l'aggiunta del sottotitolo arbitrario La televisione ha ucciso la realtà? nella traduzione del volume di Jean Baudrillard, Le crime parfait, Paris, Galilée, 1995 (Milano, Cortina, 1996).

19. Nel 1996 il prestigioso The Wall Street Journal affermava: "L'internet era un medium in cerca di un suo modello praticabile di business. Ora ne ha trovato uno: la televisione»; cit. da Franco Carlini, Lo stile del web. Parole e immagini nella comunicazione di rete, Torino, Einaudi, 1999, p. 24. 
Quella proto-televisione in fondo è rassicurante, perché presuppone che ci sia salda memoria dei pilastri di cui sarebbe l'antitesi: la cultura, la letteratura, la democrazia, la buona educazione, ecc. Quel che è peggio, tuttavia, è che si preferisce assimilare anche i nuovi media (il digitale, la rete) a quel modello per altro parziale e malinteso di televisione ${ }^{20}$. Se così fosse, basterebbero Popper e Pasolini per comprendere il presente ${ }^{21}$. Ma già quest'ultimo avvertiva che siamo di fronte a una "mutazione" antropologica»" da lui interpretata come omologazione, borghesizzazione di massa, «ideologia edonistica del consumo» ${ }^{23}$, ma pur sempre "mutazione».

Rilievi molto seri e molto preveggenti, ma molto limitati dal fatto di non avere visto il seguito, ancora peggiore. Cioè l'aprirsi delle branchie. Fuor di metafora: l'indifendibilità di una separazione dei regimi espressivi, l'impossibilità di segregare le forme simboliche (cioè i media e i loro generi) entro domini gerarchicamente prefissati e condivisi. Non si sa più, da vari decenni, se sia più importante la gastronomia o la lirica, l'animazione o la divulgazione scientifica, la finzione o il reportage, il mercato o il volontariato. Figurarsi il ruolo della "letteratura". Mentre è probabile che le gerarchie (se si vuole, i nuovi canoni) si mettano a punto tra questi regimi di senso, ai loro confini per altro osmotici e comunicanti. Una visione uni-mediale oggi è impossibile, il travaso continuo è inevitabile e necessario, e il digitale non ha una piccola parte in questo processo di trasformazione della percezione della cultura. Ciò non toglie che ci siano preferenze e differenze nell'acquisizione e nell'esperienza di questa "condizione» (per usare provocatoriamente un termine al tempo stesso kantiano e pietistico di Lyotard).

Ci sono degli imprinting mediologici che contano molto. Intendo per imprinting mediologico una forma di comunicazione che influenza più di altre la formazione di una generazione. Per fare un esempio, la generazione di Havelock, McLuhan, Lévi-Strauss, Goody, tutti studiosi che si sono posti il problema dell'oralità, ha avuto il suo imprinting con la radio.

20. Per una visione più ampia del fenomeno televisivo già alla fine degli anni Settanta si veda The New Television, a cura di D. Davis e A. Simmons, Cambridge (Mass), the MIT Press, I977.

2I. I quali proponevano in modo altrettanto paradossale e complementare o la scolarizzazione dell'apparato produttivo della televisione (Popper), o, come Ivan Illich, l'abolizione della tv e della scuola (Pasolini). Cfr. K. R. Popper, "Una patente per fare tv», in Karl Raimund Popper e John Condry, Cattiva maestra televisione, a cura di F. Erbani, Milano, Reset, 1994, alle pp. 13-25; e Pier Paolo Pasolini, "Due modeste proposte per eliminare la criminalità in Italia» (I8 ottobre 1975); "Le mie proposte su scuola e TV» (29 ottobre I975), in Lettere luterane, Torino, Einaudi, 1976, rispettivamente alle pp. I65-171 e I72-178.

22. Pier Paolo Pasolini, "IO giugno 1974. Studio sulla rivoluzione antropologica in Italia», in Scritti corsari, Milano, Garzanti, 1975, pp. 50-63, a p. 53.

23. Ivi, p. 5I. 
E poi è venuta la TV, che ha avuto un influsso più rapido e più radicale, proponendo un modello di esteriorizzazione cognitiva al tempo stesso più efficace e più vago della radio: efficace, perché delocalizzante (il suono arriva, mentre la vista implica o impone un punto di vista, un riposizionamento percettivo); vago, perché sensorialmente più completo (vista + udito + proiezione corporea + rilocalizzazione + sincronia), e quindi meno bisognoso di completamento percettivo e semantico da parte del fruitore. La nostra tradizione letteraria ha sempre dialogato proficuamente - anche se non senza qualche agonismo e conflitto - con i vecchi media come cinema e radio. Se non si vuole risalire a D'Annunzio e Pirandello, basti pensare a Zavattini, Flaiano, Soldati, allo stesso Parise ${ }^{24}$ e, soprattutto, allo stesso Pasolini.

Con più difficoltà e diffidenza si è svolto il dialogo con la televisione (forse per i pregiudizi teorici e ideologici a cui si è accennato), nonostante le molte partecipazioni (da Eco a Pasolini) e la tv "antropologica", ad esempio, di un Soldati, lungo il corso del Po, o di un Buzzati, sulle montagne. Da ultimo, tuttavia, sembra superata agilmente - pur sempre con una certa diffidenza astiosa - anche questa barriera, se si pensa a come la tv entri nell'orizzonte di romanzi come Dies Irae di Giuseppe Genna, o Troppi paradisi di Walter Siti ${ }^{25}$. Nelle rappresentazioni "letterarie" della tv sembrano spesso prevalere, comunque, o una sindrome della sparizione, come nel racconto di Juan Marsé, Il caso dello scrittore sfumato ${ }^{26}$, (dove appunto lo scrittore a contatto con la televisione "sfuma»), o una sindrome dell'alienazione, come in Farenheit 45I di Ray Bradbury (dove la tv diviene a tal punto "interattiva" da sostituirsi al reale).

Ma ciò che più importa in questo frangente, è un altro fenomeno, più che tematico e percettivo, direi, pragmatico e artistico, che deve molto al digitale. Pensiamo, tra i molti esempi che si potrebbero fare, al Vajont di Marco Paolini. Qui possiamo constatare come la circolazione intermediale possa produrre eccellenti risultati: è del 1997 (2 e 9 ottobre) la ripresa televisiva del Vajont, testo che è diventato spettacolo poi DVD e libro, e ancora TV e ancora teatro e ancora spettacolo. Michele Serra, commentando la replica TV del 27 ottobre 2003 scriveva nella sua rubrica

24. Parise, a proposito della sua prima opera, Il ragazzo morto e le comete, affermava che «La sola cultura che ha ispirato questo libro è cinematografica: ero un appassionato frequentatore di cinematografi» (Goffredo Parise, Opere, a cura di B. Callagher e M. Portello, vol. I, Milano, Mondadori, I994, p. I574).

25. Si veda, per un excursus sull'immaginario letterario italiano dei media, P. Giovannetti, Retorica dei media. Elettrico, elettronico, digitale nella letteratura italiana, Milano, Unicopli, 2004.

26. Juan Marsé, Il caso dello scrittore sfumato [I994], trad. di F. Biancatelli, Roma, Nottetempo, 2002. 
de La Repubblica, l' «Amaca» ${ }^{27}$ : «Non era "televisione", quello che guardavo, ma era in televisione che lo stavo guardando». Non è il vecchio teatro in televisione, non è la tv culturale, è qualcosa di completamente nuovo, che rompe gli argini del televisivo non verso il reality, ma verso altre più complesse realtà, verso la comunità dei viventi. Serra ribadisce, sempre nell' «Amaca»:

Rimasto di nuovo sbalordito e commosso dalla potenza rituale di uno spettacolo che è anche cerimonia funebre, denuncia civile, solenne ricomposizione - davanti all'attore celebrante - di una comunità massacrata, i vivi con i morti, i giovani con i vecchi. ${ }^{28}$

E la rete?

Credo che il punto di passaggio individuato da Baricco, negli anni Novanta, sia giusto (e questo rende ragione del mio titolo). Non credo tanto che il discrimine debba essere individuato in Google, che a mio parere è quasi una conseguenza necessaria e non inaspettata dello sviluppo precedente, ma nella diffusione della rete e nella programmazione orientata agli oggetti. Ossia quando il computer comincia a simulare l'analogico, a mostrare e manipolare oggetti e testi, a rappresentare le metafore su cui si basa l'uso sociale della rete. C'è un indizio importante che mostra questa discontinuità. Negli anni Ottanta escono molte opere la cui trama ruota intorno a un computer: ne nomino solo tre che appartengono alla fine del decennio: Codice privato, film di Francesco Maselli (1988); Il caso del computer Asia, di Gianpaolo Proni (1989); e infine Il pendolo di Foucault di Umberto Eco (1988). In tutti i casi il computer di cui si parla è una macchina primitiva, è un personal computer, pensato sostanzialmente come macchina da calcolo, non come ambiente di rete dove circolano cose, oggetti.

E lo stesso Eco, indubbiamente precoce utilizzatore del computer, pensa al computer come lo pensava la neoavanguardia: come una scatola che produce combinazioni. Il suo imprinting mediologico del computer è quello ludico-combinatorio e seriale degli anni Sessanta, condiviso per dire anche da Calvino (anche se con sfumature e differenze che meriterebbero di essere evidenziate). Un computer che aveva come risultati le poesie automatiche di Balestrini, i romanzi multipli o ricombinabili di Saporta e dello stesso Balestrini (Tristano, 1966: ne è uscita ora una riedizione

27. La Repubblica, 27 ottobre 2003 , p. I6.

28. Ibidem. 
finalmente realizzata nelle sue 2550 versioni differenti, grazie agli sviluppi del digitale) o la musica seriale di Berio. Non per nulla oggi Eco sostiene che il digitale non cambia e non aggiunge nulla a una teoria del segno; ma sarebbe come dire che la polvere da sparo non cambia nulla nella concezione della guerra.

E se leggiamo invece un racconto di Daniele del Giudice come Evil Live (1995-1996) ${ }^{29}$, vediamo subito la differenza: si parla subito di corpi, il computer veicola metaforicamente corpi di donne che lottano, suggerisce un rapporto tra corpo e macchina. Non solo, ma l'autore rende perfettamente un'altra proprietà del digitale e della rete, l'incorniciamento, la stratificazione. I corpi emergono grazie a un aumento della distanza prospettica tra cornice e narrazione di secondo e terzo grado.

Non si tratta di contaminazione di linguaggi (luogo comune della letteratura degli anni Ottanta), ma di un nuovo modo di essere attraverso $i$ linguaggi (se di "linguaggi" si tratta). Non è citazione né ibridazione, ma la consapevolezza che il cosiddetto extra-letterario non è affatto extraletterario: semplicemente diviene cornice, specola, medium, forma simbolica, strumento di connessione semantica, emotiva, sensoriale, corporea.

Anche se leggiamo una riflessione sul computer di qualche anno più tardi, dello scrittore Sandro Veronesi ${ }^{30}$, aneddotica e paradossale, ma non meno significativa, ci accorgiamo che la percezione è cambiata. Veronesi parla ancora del computer come macchina personale, ma ci si rende conto che esso è divenuto una collettività, ed è percepito come un coacervo di volontà differenti. L'attenzione non è più rivolta all'hardware, ma al software. Per Veronesi la prevista e paventata «umanizzazione» del computer sussiste in quanto esso acquisisce una profondità, magari occulta e maligna, ma che racchiude il residuo del lavoro di molti, che abitano come fantasmi il luogo dove uno scrive. L'«inconscio del computer»è in effetti la prova che esso, nella sua dimensione plurima, di rete, è venuto a far parte dell'inconscio collettivo, e che partecipa a ciò che ciascuno sente, pensa, fa e scrive.

E siamo sicuri che ciò comporti una frattura con il "letterario" di un tempo? Che sia necessariamente il preludio di un oblio della tradizione letteraria, e non piuttosto la base per un suo recupero e una sua assimilazione, quindi la premessa di una sua sostanziale innovazione? È chiaro

29. Daniele Del Giudice, «Evil Live», in Mania, Torino, Einaudi, 1997, pp. 65-83.

30. Sandro Veronesi, "L'inconscio del computer. Che cosa si nasconde dietro ai software più comuni", Corriere della Sera, II febbraio 200I, pp. I e 20; poi in Id., Superalbo. Le storie complete, Milano, Bompiani, 2002, pp. 346-349, con il titolo: I.A. L'inconscio artificiale. 
che non si può rispondere, ma è importante tenere aperta la questione per comprendere il passaggio ${ }^{3 \mathrm{I}}$.

Consideriamo ad esempio il libro di esordio di Tiziano Scarpa, Occhi sulla graticola, del $1996^{32}$.

Scarpa è impregnato di cultura classica e moderna, nella stessa misura in cui attinge a ogni genere di produzione contemporanea. Ma le citazioni non compaiono nel testo per produrre effetti comici, come sembra suggerire la seconda di copertina, non compongono un pastiche, non sono ornamento o vezzo, e nemmeno parodia: sono necessarie all'economia del racconto. Hanno una funzione regolativa, rendono il grottesco delle vicende narrate più equilibrato e più profondo. La forza di quel libro, come di altri successivi di Scarpa, non è la rottura, ma la congiunzione di un nuovo modo di sentire, forte e incontenibile, ironico e paradossale, e la tradizione. Le citazioni, apparentemente congrue come quelle di un ottimo studente di studi umanistici, riportano percezioni esasperate su un terreno comune, quello della grande letteratura, da Rabelais a Nabokov e Gadda. Solo che Scarpa sceglie un registro anti-narrativo, inventando una sorta di diario-indagine-saggio che ha come oggetti principali una donna, alla quale l'estensore del diario vorrebbe unirsi «in duraturo vincolo affettivo», e il suo presunto fidanzato. Il protagonista-estensore compila questo "rapporto" in funzione operativa (per capire come conquistare la ragazza), ma il destinatario dello scritto risulta un se stesso futuro, quindi un narratore-lettore che non c'è, che non rientra nel quadro e guarda il tutto da fuori. Anche qui, come in Del Giudice, siamo di fronte a una moltiplicazione delle cornici. Lo sdoppiamento del narratore, che viene scoperto a poco a poco dal lettore, e che produce una sospensione ironica dei punti di vista, contiene per così dire altri infiniti sdoppiamenti, che si rifrangono sia nel tessuto linguistico (bivocità o contrappunti lessicali espliciti, esposti in funzione ironica), sia nella struttura (spesso a menu), sia nei materiali eterogenei e pluriautoriali che l'estensore inserisce nel rapporto: dai commenti diretti e indiretti dell'estensore stesso sui brani inseriti (pagine di diario trafugati alla ragazza desiderata, lettere, frammenti di conversazioni, articoli, ecc.), all'intervento censorio della stessa ragazza che irrompe alla fine dello scritto e nega (negazione di sapore, come è stato detto giustamente ${ }^{33}$, vagamente sveviano) tutto ciò che precede.

31. Sull'argomento si veda l'ampia e argomentata analisi di Alberto Casadei, Stile e tradizione nel romanzo italiano contemporaneo, Bologna, Il Mulino, 2007.

32. Tiziano Scarpa, Occhi sulla graticola, Torino, Einaudi, 1996.

33. Silvana Tamiozzo Goldmann, «La bravura come limite», L’immaginazione, n. 29, 1996, p. I9. 
È probabile che Scarpa si sia ispirato consapevolmente, nella realizzazione di questo registro anti-narrativo, a un modello ipertestuale. Ciò è sottolineato anche dalla scelta di caratteri e impaginature differenti per i vari inserti e citazioni. Solo che non si tratta di una mimesi dell'ipertestualità, di una riproduzione d'accatto o del tentativo di mescidare "linguaggi", bensì di un'interpretazione, di un "esecuzione» "letteraria" di un sapere già ipertestuale e ipermediale (così come un ipertesto simula e distorce un testo, il testo simula e distorce l'ipertesto). La scrittura di Scarpa è in altre parole già all'interno di un orizzonte ipertestuale e ipermediale. L'elenco diventa anche menu e opzione, il documento inserito diviene espansione anche sinestesica di un concetto, di una percezione o di una narrazione, in funzione non definitoria o esplicativa, ma sospensiva: ogni punto di vista resta aperto a una pluralità di approfondimenti plurisensoriali e pluriautoriali. Anche qui Scarpa si rivela uno sperimentatore che manipola sapientemente la lingua, i generi, le tecniche della narrazione o dell'antinarrazione, ma nello stesso tempo fa tesoro di una tradizione novecentesca - dal futurismo a Calvino - che giunge fino alle generazioni dei padri o dei patrigni (Tabucchi, Celati) ${ }^{34}$. Già in questi autori si delinea un «riposizionamento percettivo»: costoro hanno messo in dubbio il primato della vista, hanno raccontato o mostrato nuovi tentativi di soggettività.

E nel capitolo I3 del libro di Scarpa c'è un'interessante schizzo della soggettività contemporanea, condotto giocando anche qui sulla cornice: Alfredo-estensore invita se stesso futuro e il lettore presunto, nel momento stesso in cui la propone, a non leggere una pagina del diario dell'amata, rubata e trascritta, dove questa «si rimette a sdottorare su di sé invece di raccontarci una storiella» ${ }^{35}$; "Tieni presente, Alfredo futuro, che Carolina rifrigge nel suo diario le parole-chiave che svolazzano nell'aria. Da brava vispa Teresita razzola nel praticello telelinguistico.» ${ }^{36}$ Anche qui l'espediente retorico non è puramente difensivo, perché razzolando in quei cyberluoghi comuni (la cyber-mania di cui si parlava), tra «tabù atavici» e «tubi catodici», tra "carnesangue» e "cartapesta», Scarpa attiva, spesso con effetti esilaranti, il gioco linguistico dell' «identità» contemporanea, le cui fonti fabulatorie, tematiche, ambientali sono molteplici, autenticamente multimediali: e ancora viene messa in distanza, e al tempo stesso mostrata,

34. Si veda, sul Calvino auditivo e plurisensoriale, U. Musarra-Schrøder, «Personaggi “in ascolto”. L'orecchio e l'udibile in alcuni racconti dell'ultimo Calvino», Bollettino '9oo, n. I-2, 2002, <http://www3.unibo.it/bollgoo/ numeri/2002-i/Musarra.html>.

35. Tiziano Scarpa, Occhi sulla graticola, cit., p. 59.

36. Ivi, p. 63. 
la spazzatura di cui siamo infarciti ma anche la più ampia virtù fagocitatoria, trasformativa e sperimentale della letteratura. Si trova, in Scarpa, una felice vena ironica, ma anche una altrettanto felice vena non-ironica, e questo forse è il suo valore.

Un altro indizio molto netto di una fondamentale discontinuità, prodottasi negli anni Novanta in Italia, riguarda la percezione che in quegli anni si ha della critica. Mentre i rotocalchi continuano a proporre folcloristiche mappe della critica letteraria ${ }^{37}$, escono alcuni pamphlet, interviste e interventi (anche in rete) che cominciano a parlare di «biechi recensori». A parte l'assurdità dell'etichetta (basti pensare a Oltre il Novecento di Mario Barenghi ${ }^{3}{ }^{8}$, per citare un volume che cerca di fare un consuntivo di quegli anni ed esce nel 1999, e che "bieco» non è), le argomentazioni o le lamentele degli autori di quegli scritti mostrano non solo la volontà o la sensazione di trovarsi alle soglie di un mondo nuovo, ma anche, di fatto, una mutazione irreversibile nel modo di leggere e di pensare la letteratura. E un fenomeno che indubbiamente ha le sue radici recenti negli anni Ottanta, e si attribuisce a Tondelli l'avere rilevato «la sostanziale mancanza (o quasi) di "scambi" con una critica in grado, anche "generazionalmente", di condividere con gli autori una formazione, un gusto, una "cultura" comuni» ${ }^{39}$. Ma è negli anni Novanta che si fa strada l'idea di una distanza incolmabile, soprattutto tra i critici e i nuovi lettori. La differenza, rispetto all'annoso problema dell'insufficienza della critica militante, o dello scarto generazionale, è che $\mathrm{i}$ «biechi recensori» non vengono accusati di rappresentare questo o quel potere editoriale o accademico, o di appartenere a un ceto, a un empireo qualitativo che giudica e manda, né tanto meno di appartenere a una generazione precedente, di genitori o di nonni, ma, indipendentemente dall'età e dal ruolo ${ }^{4}$, di non comprendere affatto la nuova letteratura e i suoi lettori. E il giudizio non riguarda solo chi prende

37. Si veda, tra i molti esempi che si potrebbero citare, l'organigramma in margine all'articolo dello stesso Giulio Ferroni, «Il critico muore. Di politica», Corriere della Sera, II maggio I996, p. 3I.

38. Mario Barenghi, Oltre il Novecento. Appunti su un decennio di narrativa (I988-1998), Milano, Marcos y Marcos, 1999. Altri due esempi non biechi, che mostrano la percezione forte di un cambiamento d'orizzonte della letteratura e della critica sono Emanuele Trevi, Istruzioni per l'uso del lupo. Lettera sulla critica, Roma, Castelvecchi, 1994 (Roma, Cooper \& Castelvecchi, 2002), e Guido Guglielmi, "Crisi della critica, crisi della letteratura", Bollettino 'goo, n. I-2, 200o, <http://www.unibo.it/bollgoo/numeri/200o-ii/guglielmi.html>.

39. A. Ferracuti, Presentazione a G. Picone, F. Panzieri e M. Raffaelli, Paesaggi italiani, a cura di A. Ferracuti, Transeuropa, 1994, p. I3.

40. Già Enrico Palandri scriveva, rispetto al rapporto tra generazioni: «Vi sono concordanze, linee di continuità, come pure cesure e contrasti che sono tuttaltro che generazionali. I rapporti con la lingua, con l'esperienza vissuta e con la letteratura sono lungi dal presentare un quadro compatto.» (Enrico Palandri, «Lettera a Panta», Panta, n. 5, I99I, p. I57. Corsivo mio.) 
le distanze dai nuovi narratori, o chi li condanna come prodotto infimo del tempo presente, ma anche chi li promuove, magari assimilandoli a poetiche e movimenti letterari precedenti (primo tra tutti il gruppo '63). Ë emblematico il racconto Gli orsi (63-93) ${ }^{41}$ di Silvia Ballestra, che mostra una discontinuità esistenziale e di sensibilità rispetto al mondo dei critici (fin dall'esergo leopardiano: «Se si dovessero seguire i gran principii prudenziali e marchegiani di mio padre, scriveremmo sempre sopra argomenti del secolo di Aronne») ${ }^{42}$ e al contempo di tal mondo illustra per così dire l'aggiornamento e l'inevitabilità. Anche qui accanto al grottesco trova posto il serio: la distanza di pelle e la percezione di una piccola società letteraria prescrittiva che si trasforma e si riproduce, coinvolgendo anche le nuove generazioni, e che fa tesoro delle nuove leggi del marketing casereccio e degli utilitarismi pacchiani e mediatici di oggi ${ }^{43}$.

Renzo Paris scrive:

Sono ormai tre le generazioni di scrittori che vivono da sole, senza la compagnia dei loro critici, ma negli anni Novanta spunta qualcosa di più [...]. Non è solo un conflitto nato nell'ambito del gusto. Da che mondo è mondo i vecchi se la sono presa con i giovani o mandandoli alla guerra o seviziandoli in altri modi più sottili. Il rifiuto della tribù affonda le radici nel modo di vivere dei giovani che è radicalmente cambiato nel nostro paese, attraversato dalla terza ondata del consumismo. Un tale atteggiamento [dei biechi recensori], moralisticamente preconcetto, fa il paio con quello tutto speculante delle case editrici che lanciano in modo acritico i giovani sul mercato del romanzo. Sia gli uni che gli altri non fanno i conti con la reale novità del romanzo degli anni Novanta. Il linguaggio della nuova tribù appare ostico, criptico, comunque non letterario, non facendo parte del gergo medio-colto. Chi è nato in epoca pretelevisiva ha qualche problema con la telematica e con la nuova idea di realtà con cui le nuove generazioni si confrontano o sono chiamate a creare. $\grave{E}$ l'immaginario, oltre al linguaggio, che è cambiato. ${ }^{44}$

Ferdinando Camon, che introduce un altro dei volumi usciti in quegli anni sulla nuova narrativa e sulla critica, afferma che «qui siamo nel crinale che segna la nuova generazione, o più probabilmente le nuove generazioni: non sono più generazioni endo-letterarie, hanno una formazione

4I. Silvia Ballestra, «Gli orsi (63-93)», in Gli orsi, Milano, Feltrinelli, 1994, pp. 7-29.

42. Giacomo Leopardi, Lettera a Pietro Brighenti, Recanati, 28 aprile I82o.

43. Silvia Ballestra è però più legata a un'idea di conflitto anagrafico, stando a queste sue parole: «I critici delle generazioni passate spesso non riescono a comprendere le contaminazioni tra i vari linguaggi. $\mathrm{Ci}$ sono alcuni giovani critici molto validi, però difficilmente vengono allo scoperto: non so se ciò sia dovuto a una loro precisa scelta, oppure se i cosiddetti baroni non vogliono concedere spazi alle nuove leve.» (Luca Gervasutti, Dannati \& sognatori, cit., pp. 87-88.)

44. Renzo Paris, Romanzi di culto. Sulla nuova tribù dei narratori e sui loro biechi recensori, Roma, Castelvecchi, 1995, p. 5 e pp. 7-8 (corsivo mio). Di là dal disegno tendenzioso del libretto, che tende a proporre una poetica del neo-antico, è interessante l'inchiesta su cui esso si basa. Ivi, pp. 7-8. Corsivo mio. 
extra-letteraria, ed extra-italiana» ${ }^{45}$. Anche qui, gli antecedenti diretti sono indicati da qualcuno all'inizio degli anni Ottanta, anche se poi quella linea è stata di fatto emarginata per essere ripresa negli anni Novanta. Scrivono ad esempio Fulvio Panzeri e Franco Galato: «La forza dei libri di Tondelli e Palandri è quella di porsi fuori della letteratura in senso canonico, e della tradizione italiana in genere. La parola non ha carattere estetico, ma essenzialmente emotivo e musicale.» ${ }^{46}$

Il dubbio, la sensazione di uno scarto, di uno scollamento, riguarda in fin dei conti la natura stessa della letteratura, nel momento stesso in cui i nuovi autori si ripropongono, comunque, di scrivere.

La sfida mediale alla letteratura in realtà è cominciata da più di un secolo. Ma si è intensificata quando il cinema ha conquistato le zone alte della cultura di massa, negli anni Sessanta, e ha interessato in particolare le generazioni nate allora. Andava per la maggiore nelle università italiane, ancora negli anni Ottanta, un manuale di teoria della letteratura, del 1968, che faceva finire la parabola della narrativa letteraria nelle nuove forme del cinema ${ }^{47}$. E James Ballard scriveva, nel 1990: «nel film lo scrittore deve farsi largo fra il regista, l'attore, il produttore e l'editor, che trasformano tutti insieme la parola stampata in qualcosa di gran lunga più affascinante ed evocativo.» ${ }^{8}$ Le radici di una crisi della "cultura del libro» sono antiche; ma la reazione ad essa in Italia sembra farsi strada, con maggiore consapevolezza e libertà, soprattutto negli anni Novanta, nella prassi di una parola narrativa in senso lato che deve ritrovare ab origine il proprio spazio, in primo luogo nel mondo, e solo in secondo luogo nella tradizione, come libro scritto, come "prodotto letterario". Agli occhi di queste nuove generazioni la neoavanguardia nonostante tutto è ancora, al massimo grado, libresca. E invece la consapevolezza di uno spaesamento della scrittura, della necessità di una sua ricollocazione nel complesso della cultura umana, coincide con una piccola scossa mondiale (accompagnata da altre scosse): la diffusione della rete (e la caduta del muro di Berlino, e le prime realizzazioni effettive di globalizzazione). Come scrive Lidia

45. Ferdinando Camon, Prefazione a Luca Gervasutti, Dannati \& sognatori. Guida alla nuova narrativa italiana, Pasian di Prato (UD), Campanotto Editore, I998, pp. 7-II (p. 9). Anche questo volume è interessante per la rassegna di pareri su temi specifici di scrittori e critici negli anni Novanta.

46. Fulvio Panzeri e Franco Galato (a cura di), "Cercatori di storie, videostorie e controstorie. Dieci percorsi di lettura", in Altre storie. Inventario della nuova narrativa italiana fra anni '8o e ' 90 , a cura di R. Cardone, F. Galato e F. Panzeri, Milano, Marcos y Marcos, 1996, p. 67.

47. Robert Scholes e Robert Kellogg, The Nature of Narrative, London, Oxford University press, I968; trad. it. di R. Zelocchi, La natura della narrativa, Bologna, Il Mulino, I970, e successive edizioni.

48. James G. Ballard, Fine millennio: istruzioni per l'uso [1996], trad. di A. Caronia, Milano, Baldini \& Castoldi, I999, p. I2. L'articolo citato è del 1990. 
De Federicis, «La narrativa è trasversale, nella comunicazione tecnologica e letteraria; trasversale $e$, come s'usa dire, multimediale. C'è narrazione a voce e per scritto, in versi e per figure, nei giornali e nei libri, nei fumetti, nel cinema, in televisione». Ma, aggiunge, "la forza della parola, la fabbrica del racconto di parole, in guerra o in concorrenza con gli altri mezzi, risulta uno dei fili conduttori di quell'imprevedibile divenire dei sistemi culturali in cui siamo immersi» ${ }^{49}$.

È il sentire comune di alcuni "narratori" degli anni Novanta, che tuttavia non percepiscono più una distinzione di principio tra differenti forme espressive: non rivendicano una priorità della parola scritta, anche se ne saggiano in prima istanza la praticabilità. Non si ritengono letterati, anche se sperimentano la potenza fagocitatoria e manipolatoria della letteratura. Si esercitano all'uso di una parola aperta, che attinge all'interdiscorsività non più solo verbale, ma multimodale, che fa i conti con le forme espressive con cui tutti ormai conviviamo, grazie alle proprietà diffusive, centrifughe - e non convergenti - della codifica digitale. Ciò che ne può nascere allora non è una serie di etichette (neo-barocco, postmoderno, cannibali, neo-antico, nuova epica, letteratura dell'inesperienza, post-umano, ecc.), ma una molteplice letteratura che ricomincia a parlare alle persone, delle persone, di noi, del mondo. Inventando o trascrivendo, nutrendo o nutrendosi dei più vari mezzi espressivi, facendo riferimento alla cronaca o creando un altrove. Quel che conta, in fondo, è se le opere siano buone (colpiscano, restino, diano emozioni, facciano riflettere, facciano conoscere, dilettino, si connettano alle reti del nostro immaginario), oppure no.

Non occorre farsi sociologi o critici del costume per leggere e valutare queste scritture, anche se non bastano le categorie del letterario per giudicare il letterario. È sempre stato così, in fondo, salvo brevi secoli di oblio: il "letterario" non esiste, se non al confine di tutto il resto. I cosiddetti media non sono più "temi" della letteratura o forme espressive aliene con cui una presunta "letteratura" può intrattenere rapporti di scambio, ma il tessuto stesso, l'ambiente di cui anche la parola scritta fa parte.

$\mathrm{Ci}$ vuole quella che Gabriele Frasca ha chiamato «un'attrezzata filologia dei mezzi»: studiare non solo le rappresentazioni che dei media si fanno, ma anche come essi funzionano. E se si applica questa filologia mediale, ci si accorge che il digitale è ben distante per qualità e potenzialità da tutti i media che lo hanno preceduto. Ciò si deve alla compresenza di

49. Lidia De Federicis, Prove a carico. Due anni di percorsi nella narrativa italiana, Torino, L'indice dei libri del mese, edizione fuori commercio, aprile 1997, p. 6. 
una serie di proprietà che permettono contemporaneamente immersione e riflessione, conservazione e innovazione, spettacolarizzazione e analisi, distanza e contatto ${ }^{50}$. La sua peculiarità si deve anche ad altre sue caratteristiche costituzionali: la profondità materiale dei testi (i testi digitali - di qualunque natura essi siano - essendo codificati, sono sempre testi doppi, che incorporano l'impronta di un doppio contesto); la loro stratificazione (ciò che chiamo information architecture, cioè lo strutturarsi fluido delle cornici e delle interrelazioni semantiche); la potenzialità simulatoria (cioè lo sperimentare nuove forme di astrazione - anche pragmatica - e quindi nuovi tipi di segni); l'information retrieval (Google in senso lato). Tutto ciò fa paura. Andrebbe compreso, sviluppato e abitato. Ma, come mostra Giuseppe Granieri, la tecnologia è veloce, la comprensione lenta ${ }^{51}$. Il digitale è in continuità con i mezzi precedenti solo perché li incornicia e li modifica, non perché funziona come loro: un televisore non ha la marcia indietro, come dice Willy Wonka nella Fabbrica di cioccolato, il digitale sì š.

Ma allora, tornando alla frase di Parise, forse il punto è capire che cosa si intenda per comunicazione. Siamo sicuri che ci sia una versione sola di comunicazione e sia quella televisiva, spettacolare, pubblicitaria? Siamo sicuri che lo stesso Baricco sia un interprete affidabile nella sua concezione di comunicazione, sia in linea teorica, sia nella pratica della scrittura? Dobbiamo per forza rinunciare alla riflessione, alla profondità, alla critica, all'arte e al vino buono? Lui stesso dice:

Privilegiare la comunicazione non vuol dire scrivere cose banali in modo più semplice per farsi capire: significa diventare tasselli di esperienze più ampie, che non nascono, né muoiono, nella lettura. (B, p. 96.)

Non dovrebbe scandalizzarci l'idea che un libro trovi le sue catene di senso fuori di sé, anzi! Lo diceva già Calvino: «Lo scrittore parla a un lettore che ne sa più di lui.» ${ }^{53}$ Questo è un richiamo, di fatto, a una catena di senso esterna. Ma ci sono catene e catene. Per Calvino è fondamentale la qualità. La qualità fa la differenza. C'è qualità quando un libro mette

50. Trasformando delle categorie genettiane potremmo chiamarle rispettivamente proprietà meta-discorsive e iper-discorsive. Per un approfondimento di questi aspetti e di quelli relativi alla testualità digitale rimando alla bibliografia contenuta in Federico Pellizzi, Dialogism, Intermediality and Digital Textuality, in «IasOnline. Netzkommunikation in ihren Folgen», 29 gennaio 2006, <http:/www.iasl.uni-muenchen.de/discuss/lisforen/ pellizzi_dialogism.pdf $>$.

5I. Giuseppe Granieri, Blog generation, Roma-Bari, Laterza, 2005, p. 22.

52. Il digitale è pertanto un metamedium, come lo ha definito Alan Kay ("Computer Software», Scientific American, vol. 25I, n. 3, settembre 1984, p. 54) e non un medium.

53. Italo Calvino, "Per chi si scrive? (Lo scaffale ipotetico)", in Una pietra sopra. Discorsi di letteratura e società, Torino, Einaudi, I980, p. I62. 
in gioco «la scala dei valori e il codice dei significati stabiliti» ${ }^{54}$. Quindi comunicazione non vuol dire necessariamente rinunciare alla qualità, che non è velocità come credono i barbari, ma il prendere posizione - il termine è bachtiniano - in modo credibile (incisivo) nel complesso della cultura umana. La qualità attiva catene di senso che si radicano, anche in tempi di post-human, in una comunità di senso e di relazione. Quando c'è, essa mette in gioco, parafrasando Serra, i vivi e i morti, ed ha un significato profondamente politico. Il che vuol dire: parla di noi, più che di io. Questa può essere un'interpretazione della frase di Parise. In altre parole la letteratura, se c'è, non può che essere expanded literature, per parafrasare ciò che ha scritto Gene Youngblood a proposito del cinemass. Essa si stacca dal suo medium tradizionale per poi ritornarci, ma questo suo viaggio e il come si attua sono necessari e determinanti per stabilirne il valore ${ }^{56}$.

Si può scegliere se perpetuare lo scotoma, e far finta - lasciandoci possedere dalla comunicazione così come la ha descritta Mario Perniola ${ }^{57}$ - l'indifferenziato perpetuarsi del presente - far finta, dicevo, che il mutamento e la mutazione siano favole o leggende metropolitane, e che il digitale sia un elettrodomestico come tanti. Ma in realtà il digitale non è un elettrodomestico, ma nemmeno una forma mentis astratta, è un ambiente tecno-sociale, una pragmasfera, è un punto di vista su tutti i tipi di testo, come sosteneva Espen J. Aarseth in un libro del 1997, proprio l'anno in cui nasceva Google ${ }^{58}$.

54. Ivi, p. 159.

55. Gene Youngblood, Cinema elettronico e simulacro digitale. Un'epistemologia dello spazio virtuale [1986], in R. Albertini e S. Lischi (a cura di), Metamorfosi della visione. Saggi di pensiero elettronico, Pisa, ETS, [1988] $2000^{2}$, p. 32. Il concetto è già sviluppato in Gene Youngblood, Expanded Cinema, New York, Dutton, 1969 (London, Studio Vista, 1970). Youngblood alla fine degli anni Ottanta legava il concetto di "cinema dilatato" (sostanzialmente la necessità di una ri-socializzazione di tutta la cultura visiva) al cambiamento tecnologico e sociale, percepito e non compreso, legato allo sviluppo del digitale: «Siamo turbati da impulsi nuovi e inquietanti, tentiamo di ripensare e riallineare noi stessi, e di superare la nostra tormentata sensazione di realtà nuova. Stiamo tentando di creare una nuova cultura che dia senso e dimensione umana alle tecnologie attraverso le quali ci evolviamo e dalle quali dipende il nostro futuro.» (Gene Youngblood, Cinema elettronico e simulacro digitale, cit., p. 32.)

56. Non va dimenticato inoltre che la qualità non è necessariamente prerogativa mono-autoriale. Se il ruolo dell'editing o l'insinuarsi nella produzione letteraria dell'idea teatral-cinematografica di regia, o le pluralità commerciali di Luther Blisset o Wu Ming non sono sufficienti a mostrare una dimensione pluriautorale nella letteratura, si pensi allora al ruolo delle antologie e, sopratutto delle riviste già in tutto il Novecento. Sono esempi di una pluriautoralità che non sempre si esercita all'interno di un singolo testo, ma che sempre si realizza in una pluralità di voci nel medesimo contesto.

57. Mario Perniola, Contro la comunicazione, Torino, Einaudi, 2004.

58. Espen J. Aarseth, Cybertext. Perspectives on Ergodic Literature, Baltimore, The Johns Hopkins University Press, 1997. 
